\title{
Tumor-associated macrophages: Role in the pathological process of tumorigenesis and prospective therapeutic use (Review)
}

\author{
OLGA V. ZHUKOVA ${ }^{1}$, TATIANA F. KOVALEVA ${ }^{2}$, EVGENIA V. ARKHIPOVA ${ }^{3}$ \\ SERGEY A. RYABOV ${ }^{4}$ and IRINA V. MUKHINA ${ }^{5}$ \\ Departments of ${ }^{1}$ Pharmaceutical Technology and ${ }^{2}$ Molecular and Cellular Technologies, \\ Privolzhsky Research Medical University, Nizhny Novgorod 603005; ${ }^{3}$ Pre-Clinical Research Center, \\ Central Research Laboratory, Privolzhsky Research Medical University, Nizhny Novgorod 603005; \\ ${ }^{4}$ Department of High-Molecular and Colloid Chemistry, National Research Lobachevsky State University, \\ Nizhny Novgorod 603950; ${ }^{5}$ Fundamental Medicine Institute and Physiology Department, \\ Privolzhsky Research Medical University, Nizhny Novgorod 603005, Russia
}

Received November 24, 2019; Accepted July 10, 2020

DOI: $10.3892 /$ br.2020.1354

\begin{abstract}
The aim of the present study was to evaluate the current body of knowledge regarding tumor-associated macrophages (TAMs) and their potential use in antitumor therapy, based on their role in the pathological process of tumorigenesis. For this purpose, a critical analysis of published data and summarization of the findings available from original studies, focusing on the role of TAMs in the pathological process, and their potential therapeutic application was performed. Promising key avenues of research were identified in this field. The following issues seem the most promising and thus worth further investigation: i) The process of M1/M2 macrophage polarization, macrophage characteristics at intermediate polarization steps and their role in the tumor process; ii) determining the conditions necessary for transitions between the M1 and M2 macrophage phenotypes and the role of signals from the microenvironment in this process; iii) cause-and-effect associations between the quantity and quality of macrophages, and the prognosis and outcome of the pathological process; iv) modulation of macrophages and stimulation of their phagocytic activity with drugs; v) targeted vector-based systems for drug delivery to macrophages; and vi) targeted drug delivery systems with macrophages as carriers, thus potentially combining chemotherapy and immunotherapy.
\end{abstract}

Correspondence to: Professor Olga V. Zhukova, Department of Pharmaceutical Technology, Privolzhsky Research Medical University, Minin and Pozharsky Square, 10/1, Nizhny Novgorod 603005, Russia E-mail: ov-zhukova@mail.ru

Key words: macrophages, tumor-associated macrophages, M1/M2 polarization, tumor microenvironment, targeted drug delivery systems, chemotherapy, immunotherapy

\section{Contents}

1. Introduction

2. TAMs: General characterization

3. Prospective use of TAMs for anticancer therapy

4. Systems for targeted drug delivery to TAMs

5. Macrophages as carriers of anticancer drugs: Integration of chemotherapy and immunotherapy to target tumorigenesis

6. Conclusions

\section{Introduction}

The incidence of cancer is continuously increasing (1-8). Cancer is the second leading cause of death after cardiovascular disease worldwide, accounting for an estimated 9.6 million deaths, or one in six deaths in 2018 (9). A number of highly effective drugs are available for modern pharmacotherapy; however, the results are often unsatisfactory. Cancer treatments are associated with severe side effects, high toxicity, poor pharmacokinetics properties, lack of water solubility, lower therapeutic indices, and development of drug resistance $(4,5,10-12)$. Unfavorable biodistribution is one of the primary factors that reduce drug efficacy and may arise when drug penetration into the desired pathological site is hindered. The drug dose is frequently increased to overcome this hindrance and to improve the treatment efficacy. Hence, a therapeutic effect is often achieved at the cost of higher non-specific toxicity. The problem is particularly pressing in the case of anticancer drugs, the side-effects of which are severe enough to substantially reduce the therapeutic value of the drugs. Novel therapeutic means are continuously sought out in view of this, and immunotherapy is among the most promising avenues. Following the discovery of alternative pathways of macrophage activation, specific attention has been paid to tumor-associated macrophages (TAMs), their roles in the pathological process of tumorigenesis, and the possibility of their use in anticancer therapy. During the writing of this 
review, the most recent studies in the field of TAMs were analyzed, and the possibility of using macrophages as a point for therapeutic exposure was considered; in such a structure, a review is presented for the first time, to the best of our knowledge.

A feature of the present review is the systemized presentation of cancer treatment directions aimed at macrophages, the process of their polarization, and their therapeutic use. Three primary strategies are described: i) Drugs that are able to modulate the activity of TAMs; ii) designed carriers for targeted drug delivery to macrophages, TAMs or specific pro-tumor M2-TAMs; and iii) the use of macrophages to target the tumor. At present, research regarding TAMs is largely focused on an increased interest in the search for markers characterizing functionally different subpopulations of macrophages associated with tumor progression and the effectiveness of chemotherapy, which may result in identification of potential targets for treatment (13-18). The simplified dichotomous classification of M1/M2 provides a conceptual basis for describing the polarization of macrophages and the identification of polarizing stimuli (19-24). The high plasticity of macrophages with respect to changes in their polarization under the influence of various microenvironmental conditions opens up prospects for the directed differentiation of macrophages into an antitumor M1 phenotype or blocking of M2 polarization.

The objectives of the present review were to assess the state of TAM research and to evaluate the possible use of TAMs in cancer therapy.

\section{TAMs: General characterization}

The process of tumorigenesis in the body begins to affect the tumor microenvironment, including macrophages. Blood monocytes penetrate the tumor, and differentiate into macrophages with an anti-inflammatory phenotype in response to signaling molecules produced by the tumor, such as interleukin (IL)-4, IL-10 and transforming growth factor (TGF)- $\beta$. These signals suppress antitumor immunity, and stimulate the development of new blood vessels and thus tumor growth and metastasis (17). The role of TAMs in tumor progression are illustrated in Fig. 1.

TAMs have attracted substantial attention for the past 30 years (from the time when the concept of a macrophage dichotomy was advanced) (25,26). TAMs are classed as type II-activated macrophages (M2). Stein et al (27) first characterized TAMs as alternatively activated macrophages in 1992. Data on TAM markers and TAM-suppressing factors subsequently accumulated in further studies (28-31). The M2 population is highly heterogeneous $(32,33)$. Macrophages with the M2 phenotype serve an important role in the process of tumorigenesis by suppressing the immune response, remodeling the extracellular matrix and stimulating angiogenesis (26). M2 macrophages are characterized by the expression of specific receptors, such as arginase-1, mannose receptor (CD206), CD163, CD209, FIZZ1 and Ym1/2 (22,29).

Macrophages with the M1 phenotype (classically activated macrophages) express bactericide molecules and receptors (34). Macrophages acquire the M1 phenotype in response to endogenous inflammatory stimuli, such as the Th1-associated cytokine interferon- $\gamma$, or exogenous stimuli, such as lipopolysaccharides (23,35). M1 macrophages produce pro-inflammatory cytokines and thereby stimulate the inflammatory response (36). A total of 5,598 publications on TAMs were available on PubMed as of July 10, 2020. The annual number of such publications increased from 51 in 2007 to 660 in 2019.

Macrophages are intricately involved in the immune response, and thus serve a protective role. They participate in the clearance of cellular debris and iron processing, degradation of dead cells and foreign material, response to infection, immunomodulation and modulation of inflammatory processes, angiogenesis, and facilitating wound healing $(20,22,23)$. Furthermore, macrophages serve an important in organ development, and in tissue turnover and regeneration (37,38). Adverse reactions are also often caused by macrophages and are associated with their M1/M2 polarization. M1 macrophages serve critical roles in innate host defense and in the killing of tumor cells. Therefore, they are considered as antitumor macrophages. M2 macrophages tend to exert an immunosuppressive phenotype, favoring tissue repair, and tumor promotion. Thus, they are considered as pro-tumorigenic macrophages. The expression of inhibitory cytokines in tumor cells or macrophages provides a mechanism of resistance to anticancer therapy. Hence, a therapeutic strategy targeting macrophages or macrophage-derived cytokines may be a promising and effective method for targeting tumorigenesis (39).

TAMs are an important component of the tumor microenvironment, which affects tumor growth, tumor angiogenesis, immunity suppression, metastasis and chemoresistance. TAMs substantially affect the clinical efficacy of these drugs and drug resistance. For example, TAMs release chemoprotective factors, such as cathepsin b and milk-fat globule EGF-VIII, which promotes tumorigenicity of cancer stem cells and induces anticancer drug resistance. Furthermore, drugs targeting TAMs have been shown to exhibit promising results for potential use in anticancer therapy (40). The role that macrophages serve in carcinogenesis has been the focus of several studies, including systematic reviews (41-43).

M1 macrophages promote tumor elimination, whereas M2 macrophages facilitate carcinogenesis (44). As demonstrated over half a century ago, M1 macrophages are capable of killing and eliminating cancer cells in accordance with their primary physiological function, the elimination of foreign and harmful substances (45). M1 cells initiate cytokine production in the tumor microenvironment and facilitate cancer cell destruction by recruiting pro-immunostimulatory leukocytes and phagocytizing tumor cells $(46,47)$. M2 macrophages serve a leading role in tumor spread (48). M2 macrophages have a notable effect on tumor development in both the primary and metastatic foci. Their effects are associated with basement membrane degradation, angiogenesis and general immunosuppression (49,50). Macrophages have been shown to be present not only in the M1 or M2 states in the tumor microenvironment, but also in transitional states, and the role of the transitional states in tumorigenesis remains poorly understood (51). The elimination of all macrophage populations regardless of the polarization state may provide a potentially effective approach to therapy as both primary and metastatic tumorigenesis is reduced as a result (52). 


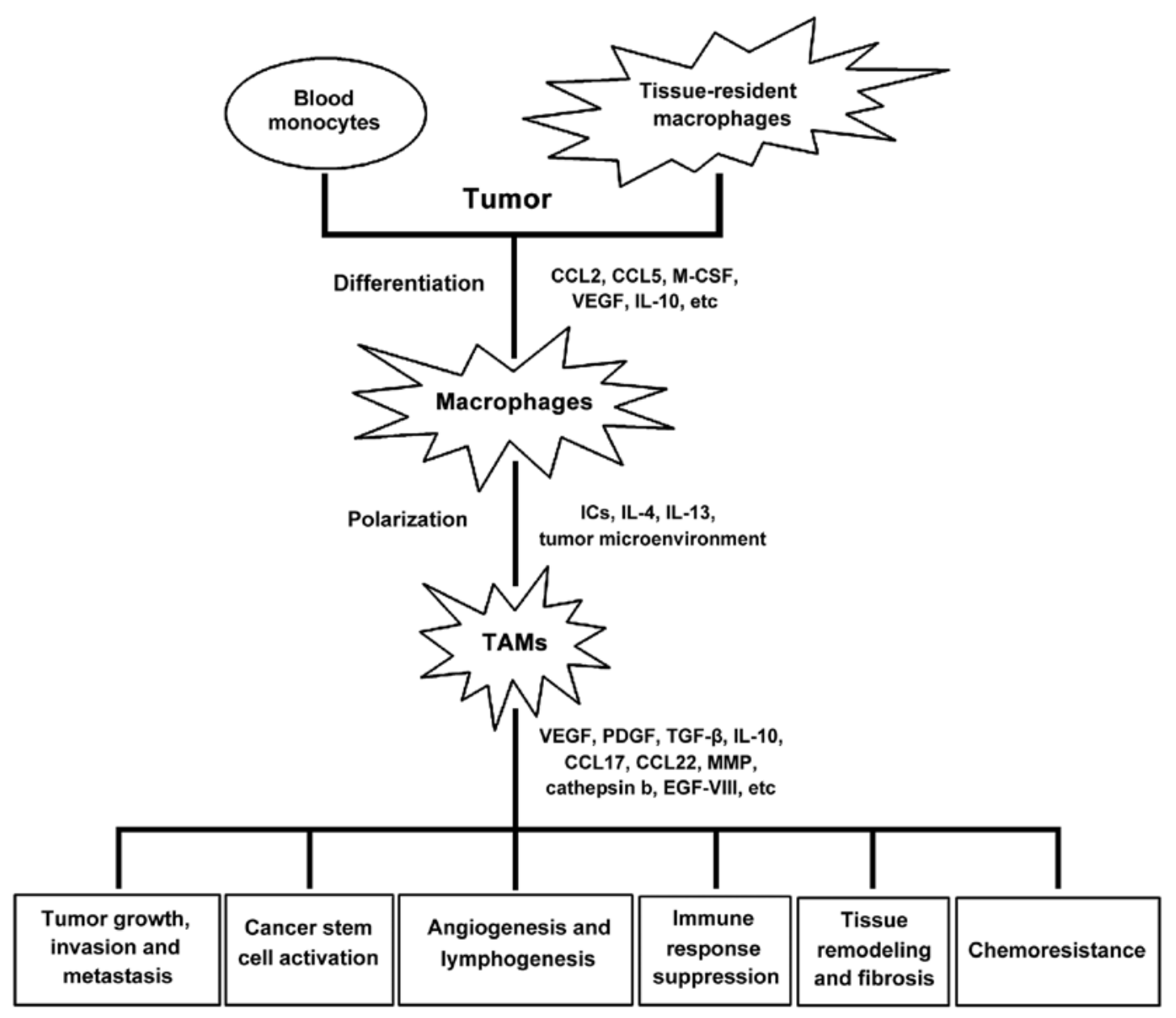

Figure 1. Cellular origin and the role of TAMs in tumor progression. CCL, C-C motif ligand; EGF-VIII, epidermal growth factor; ICs, immunocomplexes; IL, interleukin; M-CSF, macrophage-colony stimulating factor; PDGF, platelet-derived growth factor, TGF- $\beta$, transforming growth factor- $\beta$; TAMs, tumor-associated macrophages; VEGF, vascular endothelial growth factor; MMP, matrix metalloproteinase.

The activation of macrophages is widely regarded as polarization in the direction of the M1 or M2 states. However, the M2 activation state includes heterogeneous and functionally distinct macrophages. Studies on the existence of macrophages of the M2a, M2b, M2c and M2d phenotypes make it possible to specify a number of aspects regarding the nature of the immune response (Table I).

M2a and M2b phenotype macrophages typically exhibit anti-inflammatory activity. Macrophages of the M2c phenotype are very similar to M1 macrophages, with the exception of high (increased) IL-10 expression as opposed to pro-inflammatory cytokines (53). Wang et al (54) isolated the M2d phenotype, characterized by decreased secretion of IL-12 and increased secretion of IL-10. M2d macrophages are common in the tumor microenvironment. It is hypothesized that M2d macrophages are induced following stimulation with Toll-like receptor agonists and adenosine, and/or tumor-related factors. Isolation of subtypes of macrophages of the M2 family may facilitate the possibility of their targeted therapeutic use for treatment of tumors.

However, Quail and Joyce (55) demonstrated that the clinical effect of isolation of subtypes of macrophages of the M2 family was limited due to the limitations in the methods of targeted drug delivery to macrophages. However, macrophages preserve their plasticity regardless of polarization and, in particular, remain capable of switching from one phenotype to another dependent on the stimuli from the microenvironment (55).

The presence of macrophages in primary tumors is associated with a poor prognosis (56-59), with colorectal cancer serving as the only exception (60). M1 and M2 macrophages present in the tumor microenvironment have been the focus of an increasing number of studies $(20,28,51)$. Although the causal associations have not yet been established, the available body of research highlight the possibility of novel therapeutic strategies that are aimed at eliminating macrophages or altering the macrophage phenotype (61).

Since increased TAM infiltration is associated with a poor prognosis and therapeutic failure in cancer, TAM reprogramming toward the anticancer M1 phenotype and TAM suppression may provide promising strategies for the treatment of cancer (62).

\section{Prospective use of TAMs for anticancer therapy}

Based on the literature search performed for the present review, three macrophage-related strategies of cancer therapy are speculated. These strategies involve drugs that modulate TAM activity; engineered carriers for targeted drug delivery to macrophages, TAMs, or specific pro-tumoral M2-TAMs; and macrophage self-targeting to the tumor. 
Table I. Classification of phenotypes of M2 macrophages.

Phenotype of

M2 macrophages

Stimulus for polarization

Produced cytokines

Functions

\begin{tabular}{|c|c|c|c|}
\hline M2a & IL-4, IL-13 & $\begin{array}{l}\text { IL-10, TGF- } \beta \text {, IL-1 receptor } \\
\text { antagonist }\end{array}$ & $\begin{array}{l}\text { Activation of Th2-reactions, } \\
\text { eosinophil involvement, } \\
\text { connective tissue growth }\end{array}$ \\
\hline $\mathrm{M} 2 \mathrm{~b}$ & IL-1 $\beta$, immunocomplex + lipopolysaccharide & IL-1, IL-6, IL-10, TNF- $\alpha$ & $\begin{array}{l}\text { Regulation of inflammatory } \\
\text { and immune responses, } \\
\text { activation of Th2 }\end{array}$ \\
\hline $\mathrm{M} 2 \mathrm{c}$ & IL-10, TGF- $\beta$, glucocorticosteroids & IL-10, TGF- $\beta$ & $\begin{array}{l}\text { Remodeling, intercellular } \\
\text { matrix synthesis }\end{array}$ \\
\hline M2d & IL-6, adenosine & IL-10, IL-12, TNF- $\alpha$, TGF- $\beta$ & $\begin{array}{l}\text { Tumor metastasis and } \\
\text { progression }\end{array}$ \\
\hline
\end{tabular}

IL, interleukin; TGF- $\beta$, transforming growth factor- $\beta$; TNF- $\alpha$, tumor necrosis factor- $\alpha$.

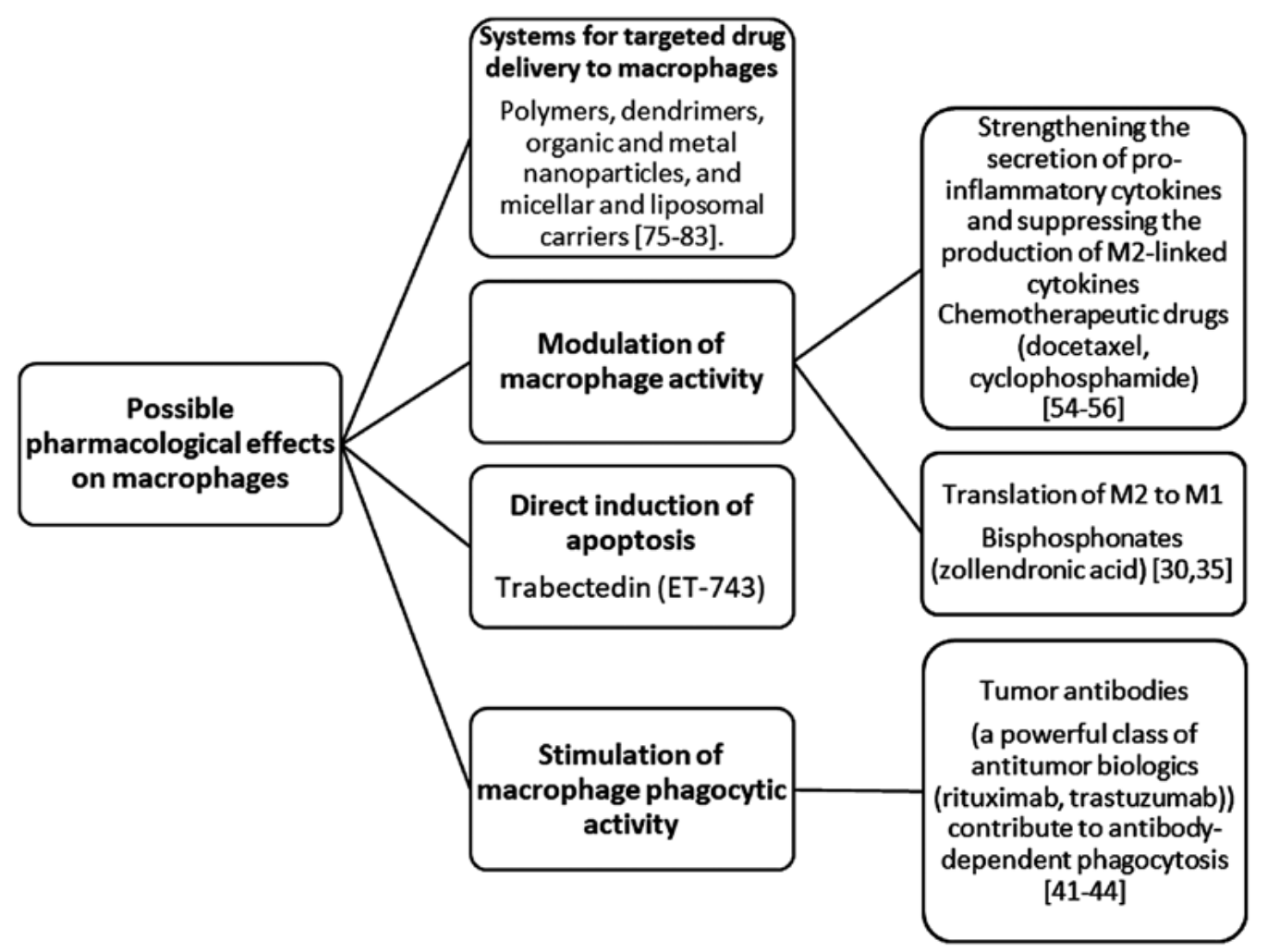

Figure 2. Potential directions of pharmacological influence on macrophages in the treatment of the tumor process

Drugs modulating macrophage activity. Various drugs that modulate macrophage activity are illustrated in Fig. 2.

Bisphosphonates modulate macrophage activity and are used in the treatment of bone tissue disorders, such as osteoporosis and bone metastases in cancer. A previous preclinical study using a mouse model of breast tumors suggested that an extra skeletal therapeutic effect is additionally exerted by bisphosphonates (63).

Zoledronic acid, which is a medication used in the treatment of cancer, has been shown to revert macrophage polarization from the M2 to the M1 phenotype, thus inhibiting spontaneous breast carcinogenesis (64). Zoledronic acid acts as a potent inhibitor of farnesyl pyrophosphate synthase, which is a key enzyme of the mevalonate pathway. By inhibiting farnesyl pyrophosphate synthase, zoledronic acid prevents the prenylation of small G-proteins, such as Ras, Rho and Rap1A, which are necessary for cancer cell adhesion, migration and invasion. It has been shown that zoledronic acid binds primarily with microcalcifications present in breast tumors and is then phagocytized by TAMs, leading to apoptosis and M2-to-M1 transformation. It has been demonstrated in vivo that zoledronic acid inhibits the production of the proangiogenic factor, matrix metalloproteinase, and triggers the TAM transition from the pro-tumoral M2 phenotype to the antitumor M1 phenotype (65). 
In 2013, Rogers (66) examined the antitumor effects of zoledronic acid in vitro and in vivo by evaluating its effect on macrophages. J774 macrophages were treated with zoledronic acid alone and in combination with doxorubicin in vitro and apoptosis and necrosis were evaluated. Following treatment with zoledronic acid, its uptake was estimated by detecting unprenylated Rap1A (uRap1A) in $\mathrm{J} 774$ macrophages in vitro, and in peritoneal macrophages and macrophage populations from subcutaneous breast cancer xenografts in vivo. The treatment of $\mathrm{J} 774$ macrophages with $5 \mu \mathrm{M}$ zoledronic acid for $24 \mathrm{~h}$ significantly increased the uRap1A levels, while apoptotic cell death was induced at higher concentrations or longer exposure times. Doxorubicin (10 nM, $24 \mathrm{~h})$ and zoledronic acid (10 $\mu \mathrm{M}$, $24 \mathrm{~h}$ ) used consecutively increased cell death compared with that observed with the use of either drug alone. Detectable uRap1A levels were observed in peritoneal macrophages and macrophage populations isolated from breast tumor xenografts $24 \mathrm{~h}$ after single administration of zoledronic acid at $100 \mu \mathrm{g} / \mathrm{kg}$ in vivo (66). Zoledronic acid concentrations $<10 \mathrm{nM}$ were shown to inhibit Rab prenylation in $\mathbf{J} 774$ macrophages following long-term exposure in culture. Quantitative mass spectrometry identified 18 different unprenylated Rab proteins and revealed that their accumulation increased at least 7-fold following the treatment of J774 cells with nanomolar concentrations of zoledronic acid (67).

Another study demonstrated that zoledronic acid combined with ultrasonic treatment was significantly more effective than zoledronic acid alone $(\mathrm{P}<0.01)$. The $\mathrm{B} 02$ tumor size in mice treated with zoledronic acid and ultrasound was $42 \%$ lower $(\mathrm{P}<0.002)$ compared with mice treated with bisphosphonate alone (68). Bisphosphonates are administered in liposomes or attached to nanoparticles to improve their pharmacokinetics, reduce the side-effects and to alter their biodistribution (65). Liposomal bisphosphonate forms are capable of inducing the M2-to-M1 phenotypic transition (69).

Thus, studies on bisphosphonates used alone or in combination with anticancer drugs or physicochemical methods for the treatment of tumorigenesis are promising fields of research, and highlight possibility of developing novel therapeutic strategies (70-74).

Drug-dependent stimulation of phagocytic activity to modulate macrophages. The phagocytosis of foreign bodies, apoptotic cells and cancer cells, and the stimulation of adaptive immunity by presenting the antigens of assimilated materials, are two important innate immune functions of macrophages (75). Tumor-specific antibodies are a class of potent biopharmaceuticals, which act by directly inhibiting the transmission of survival signals, mediating antibody-dependent cell cytotoxicity of natural killer cells, inducing complement-dependent cytotoxicity via the activation of the complement cascade, and thus promoting antibody-dependent cell phagocytosis by macrophages (76). Studies have indicated that monoclonal antibodies approved as anticancer drugs, such as rituximab and trastuzumab, exert their therapeutic effects mostly through antibody-dependent cell phagocytosis $(77,78)$. In spite of their potential to stimulate tumor cell invasion, TAMs are capable of tumor cell phagocytosis in the presence of target antibodies $(79,80)$.

Thus, to improve the therapeutic strategy based on stimulating antibody-dependent cell phagocytosis, the Fc fragments of antibodies should be engineered to increase their interaction with receptors on macrophages (81). Although IgG class antibodies are typically used to design antibody-based therapeutics, the therapeutic potential of other antibody isotypes ( $\operatorname{Ig} \mathrm{A}$ and $\operatorname{IgE}$ ) has been the subject of several preclinical studies, where monocytes/macrophages also serve an important role in affecting the functions of antibody-dependent cell cytotoxicity and antibody-dependent cell phagocytosis $(82,83)$.

Chemotherapeutic drugs are also considered potential means with which to modulate macrophages. A number of anticancer chemotherapeutics exert their pharmacological effects on non-tumor cell populations, although additional studies are required in the field, as the current literature is limited to preliminary results from in vitro experiments (84-89) In particular, trabectedin and lurbinectedin (a second-generation analog) are efficient in eliminating TAMs $(84,85)$. Trabectedin mechanically interacts with the TRAIL-R2 ligand-receptor, and induces tumor necrosis factor-related apoptosis of mononuclear phagocytes by causing receptor clustering and subsequent caspase 8-dependent apoptosis activation (86).

In addition to exerting cytotoxic effects, certain chemotherapeutics modulate the macrophage response to the tumor $(15,87)$. A previous study using mouse models of fibrosarcoma and breast tumors demonstrated that docetaxel promotes target cell polarization to macrophages with an antitumor M1 phenotype (88). Cyclophosphamide treatment facilitates macrophage infiltration, increases the secretion of proinflammatory cytokines (IL-6 and IL-12) and suppresses the production of pro-tumoral M2-associated cytokines (IL-4, IL-10 and IL-13) (89,90). As a mechanism of self-protection against chemotherapy, chemoresistant cancer cells secrete IL-34, which increases their survival and promotes the polarization of TAMs towards an M2 phenotype to further facilitate an immunosuppressive environment (91). Thus, a combination of chemotherapy and immunotherapy may be more efficient in inducing tumor regression.

\section{Systems for targeted drug delivery to TAMs}

Systems for targeted drug delivery to TAMs are associated with the second strategy of the macrophage-related therapy of cancer. After establishing the positive effects of a drug, the next focus of research should be to determine strategies with which to selectively deliver the drugs to TAMs with minimal side-effects on healthy cells (92-97).

Phagocytic activity is extremely high in macrophages. Microparticles and nanoparticles are efficiently phagocytized by macrophages. However, the rate of phagocytosis is influenced by certain properties of micro- and nanoparticles, such as the shape, size, contact angle and surface charge (98-100). Liposomes are captured by macrophages more rapidly and in greater quantities when their size is increased to $>100 \mathrm{~nm}$, particularly when $1-3 \mu \mathrm{m}$ in size. A decrease in liposome size to $<100 \mathrm{~nm}$ similarly increases their capture by macrophages (101). The composition and structure of particles also affects their capture by macrophages $(102,103)$.

Particles with highly positive or highly negative $\zeta$ potentials are captured with improved efficiency by macrophages compared with particles having a nearly neutral $\zeta$ 
potential. Spherical particles are captured more efficiently than cylindrical particles (104).

There is still no universal method available to ensure specific molecular targeting to TAMs. In 2013, Cieslewicz et al (105) reported the discovery of the so-called M2pep peptide sequence. The M2pep preferentially binds to mouse M2 cells, including TAMs, and exhibits a low affinity for other cells. Confocal visualization revealed that M2pep accumulated in TAMs in vivo after being injected into the tail vein of mice. The injection of M2pep with a pro-apoptotic peptide into the tail vein increased mouse mortality, and selectively reduced the M2-like TAM population. The study by Cieslewicz et al (105) was among the first to describe a molecular targeting construct for mouse TAMs, supporting the targeted approach to cancer therapy.

Cancer immunotherapy aimed at selectively modulating M2-like TAMs and enabling the reversal of the M2-to-M1 ratio is a promising therapeutic strategy. In 2017, Ngambenjawong and Pun (106) reported the construction of a high-avidity macrophage-selective drug delivery platform on the basis of M2 macrophage-targeting peptides (M2pep) grafted on poly(N-(2-hydroxypropyl)methacrylamide). Furthermore, polymer-grafted M2pep exhibited increased serum stability in addition to increased M2 macrophage-selective toxicity (106).

A targeted system was constructed using a copolymer of hyaluronic acid with poly(lactic acid) and poly(glycolic acid). The copolymer was assembled together with the anticancer drug, SN38, in an aqueous phase, and the nanoparticle surface was then coated with methoxypoly(ethylene glycol)-b-poly(histamine methacrylamide) via hydrophobic association to improve the colloid stability both in vitro and in vivo. When the nanoparticles arrived into the tumor microenvironment, which is acidic, the coating was detached from the nanoparticle surface as a result of the c harge transition of the poly(histamine methacrylamide) blocks from a neutral hydrophobic to a positively charged hydrophilic state through an acid-acid state, which was induced by the protonation of the imidazole groups in the tumor microenvironment (an acidic medium). The exposure of the nanoparticle shell led to an increased uptake of nanoparticles by CD44-expressing tumor cells, including cancer cells and TAMs (107).

Nanosystems can rationally be designed to attain multivalent states and, when necessary, multifunctional entities with multiplex and/or enhanced biological activity. Nanosystems engineered to contain macrophage-specific targeting fragments and loaded or associated with drugs are promising options for modulating or even eliminating pro-tumoral macrophages in vivo (108). Engineered nanosystems include polymers, dendrimers, organic and metal nanoparticles, and micellar and liposomal carriers (109-117).

\section{Macrophages as carriers of anticancer drugs: Integration of chemotherapy and immunotherapy to target tumorigenesis}

Taking advantage of the ability of macrophages to target and migrate to the tumor is the third promising strategy involving the use of macrophages. Macrophages have attracted substantial interest as carriers for drug delivery. This is due to their ability to target the tumor, their high phagocytic activity toward drug-loaded nanoparticles and their capability to directly kill cancer cells (118). For example, peritoneal macrophages can be loaded with drugs, typically included in nanoparticles or liposomes, and can then be transferred back to animals or patients $(119,120)$. Another approach that takes advantage of macrophage self-targeting to the tumor is the in vivo administration of nanoparticles of a suitable size with a specific ligand to allow nanoparticle uptake by macrophages or TAMs, and subsequent prolonged release (121-123). The long-term survival of the macrophage host is limited by the toxicity of the drug. It is thus advisable to use systems for drug delivery to decrease the acute toxicity to the macrophage carrier. When the drug is not toxic to macrophages, a proper formulation ensures the prolonged release of the loaded drug from macrophages for at least two weeks, as demonstrated by Dou et al $(124,125)$ for indinavir associated with nanoparticles.

With the appropriate strategy for nanoparticle encapsulation to ensure intracellular stability, biological preparations, such as proteins, can be loaded in macrophages $(126,127)$. Chang et al (101) demonstrated that the size of nanoparticles internalized in macrophages may substantially affect their macrophage uptake. Smaller nanoparticles (30-50 nm) exhibited increased macrophage uptake compared with larger nanoparticles (100-500 nm), but this reduced the macrophage migration velocity at the same time. Nanoparticles with a size of $100 \mathrm{~nm}$ were shown to provide a good balance between efficient drug loading and macrophage migration (101).

To investigate the pharmacological activity of carriers captured by macrophages, macrophages have been loaded with temperature-sensitive liposomes for inducible release (121), nanosized silica-gold nanoshells for photothermal therapy (120) and iron oxide nanoparticles $(119,128,129)$.

In 2015, Miller et al (122) described the use of polymeric nanoparticles, that included a platinum (IV) prodrug and a clinically tested carrier based on a copolymer of poly(lactic acid) and poly(glycolic acid) with polyethylene. The nanoparticles were shown to facilitate the long-term circulation of the drug and its uptake in TAMs. The simultaneous visualization of the carrier and its useful load with the drug revealed that TAMs serve as a drug depot and accumulate substantial quantities of the carrier, which gradually releases platinum to damage DNA in the neighboring cancer cells (122).

The reduction of TAM survival is generally considered to improve the therapeutic effects of anticancer therapy. The direct induction of apoptosis with chemical or synthetic substances provides an efficient strategy with which to eliminate TAMs. Trabectedin (ET-743) is an anticancer drug used in the treatment of platinum-sensitive soft-tissue sarcomas. The drug causes selective TAM depletion in cancer patients by activating the extrinsic apoptotic pathway through TRAIL receptors. As trabectedin directly affects monocyte/macrophage-mediated host defense in addition to targeting TAMs (85), designing TAM-specific agents may result in a reduction of side-effects.

\section{Conclusions}

Reviewing TAM-related literature revealed that the number of publications in this field has increased in number over the past three years, highlighting novel possibilities for the use of a combination of immunotherapy and chemotherapy to 
treat cancer. However, there are several issues which warrant further investigation. These issues include: Attaining a deeper understanding of the process of M1/M2 macrophage polarization, macrophage characteristics at intermediate polarization steps, and their role in tumorigenesis; the conditions that are necessary for transitions between the M1 and M2 macrophage phenotypes and the signals that this process is dependent on from the microenvironment; the cause-and-effect relationships between the quantity and quality of macrophages, and the prognosis and outcome of the pathological process; modulation of macrophages and stimulation of their phagocytic activity with drugs; development of suitable and safe targeted vector-based systems for drug delivery to macrophages; and the development of targeted drug delivery systems with macrophages as carriers, thus potentially combining chemotherapy and immunotherapy.

\section{Acknowledgements}

Not applicable.

\section{Funding}

No funding was received.

\section{Availability of data and materials}

Not applicable.

\section{Authors' contributions}

OVZ, IVM, TFK, EVA and SAR were all involved in drafting and revising the manuscript. All authors read and approved the final manuscript.

\section{Ethics approval and consent to participate}

Not applicable.

\section{Patient consent for publication}

Not applicable.

\section{Competing interests}

The authors declare that they have no competing interests.

\section{References}

1. Bray F, Ferlay J, Soerjomataram I, Siegel RL, Torre LA and Jemal A: Global cancer statistics 2018: GLOBOCAN estimates of incidence and mortality worldwide for 36 cancers in 185 countries. CA Cancer J Clin 68: 394-424, 2018.

2. DeSantis CE, Lin CC, Mariotto AB, Siegel RL, Stein KD, Kramer JL, Alteri R, Robbins AS and Jemal A: Cancer treatment and survivorship statistics, 2014. CA Cancer J Clin 64: 252-271, 2014.

3. Hanahan D and Weinberg RA: The hallmarks of cancer. Cell 100: $57-70,2000$

4. Mitra AK, Agrahari V, Mandal A, Cholkar K, Natarajan C Shah S, Joseph M, Trinh HM, Vaishya R, Yang X, et al: Novel delivery approaches for cancer therapeutics. J Control Release 219: 248-268, 2015.
5. Talekar M, Tran TH and Amiji M: Translational nano-medicines: Targeted therapeutic delivery for cancer and inflammatory diseases. AAPS J 17: 813-827, 2015.

6. Torre LA, Bray F, Siegel RL, Ferlay J, Lortet-Tieulent J and Jemal A: Global cancer statistics, 2012. CA Cancer J Clin 65: 87-108, 2015.

7. You W and Henneberg M: Cancer incidence increasing globally: The role of relaxed natural selection. Evol Appl 11: 140-152, 2017.

8. Zhang Z, Zhou L, Xie N, Nice EC, Zhang T, Cui Y and Huang C: Overcoming cancer therapeutic bottleneck by drug repurposing. Signal Transduct Target Ther 5: 113, 2020.

9. World Health Organization (WHO): Cancer: Key facts. WHO, Geneva, 2018. https://www.who.int/news-room/fact-sheets/ detail/cancer. Accessed September 12, 2018.

10. Lewis LD: Cancer pharmacotherapy: 21 st century 'magic bullets' and changing paradigms. Br J Clin Pharmacol 62: 1-4, 2006.

11. Samadi AK, Bilsland A, Georgakilas AG, Amedei A, Amin A, Bishayee A, Azmi AS, Lokeshwar BL, Grue B, Panis C, et al: A multi-targeted approach to suppress tumor-promoting inflammation. Semin Cancer Biol 35 (Suppl): S151-S184, 2015.

12. Shaked Y: The pro-tumorigenic host response to cancer therapies. Nat Rev Cancer 19: 667-685, 2019.

13. Guo Q, Jin Z, Yuan Y, Liu R, Xu T, Wei H, Xu X, He S, Chen S, Shi Z, et al: Corrigendum to 'New mechanisms of tumor-associated macrophages on promoting tumor progression: Recent research advances and potential targets for tumor immunotherapy'. J Immunol Res 2018: 6728474, 2018.

14. Larionova I, Cherdyntseva N, Liu T, Patysheva M, Rakina M and Kzhyshkowska J: Interaction of tumor-associated macrophages and cancer chemotherapy. Oncoimmunology 8: 1596004, 2019.

15. Mantovani A and Allavena P: The interaction of anticancer therapies with tumor-associated macrophages. J Exp Med 212: 435-445, 2015.

16. Mantovani A, Marchesi F, Malesci A, Laghi L and Allavena P: Tumour-associated macrophages as treatment targets in oncology. Nat Rev Clin Oncol 14: 399-416, 2017.

17. Nielsen SR and Schmid MC: Macrophages as key drivers of cancer progression and metastasis. Mediators Inflamm 2017: 9624760, 2017.

18. Prill S, Rebstock J, Tennemann A, Körfer J, Sönnichsen R, Thieme R, Gockel I, Lyros O, Monecke A, Wittekind C, et al: Tumor-associated macrophages and individual chemo-susceptibility are influenced by iron chelation in human slice cultures of gastric cancer. Oncotarget 10: 4731-4742, 2019.

19. Lampiasi N, Russo R and Zito F: The alternative faces of macrophage generate osteoclasts. Biomed Res Int 2016: 9089610, 2016.

20. Lin Y, Xu J and Lan H: Tumor-associated macrophages in tumor metastasis: Biological roles and clinical therapeutic applications. J Hematol Oncol 12: 76, 2019.

21. Mantovani A, Sica A, Sozzani S, Allavena P, Vecchi A and Locati M: The chemokine system in diverse forms of macrophage activation and polarization. Trends Immunol 25: 677-686, 2004.

22. Porta C, Riboldi E, Ippolito A and Sica A: Molecular and epigenetic basis of macrophage polarized activation. Semin Immunol 27: 237-248, 2015.

23. Schliefsteiner C, Peinhaupt M, Kopp S, Lögl J, Lang-Olip I, Hiden U, Heinemann A, Desoye G and Wadsack C: Human placental hofbauer cells maintain an anti-inflammatory M2 phenotype despite the presence of gestational diabetes mellitus. Front. Immunol 8: 888, 2017.

24. Yao Y, Xu XH and Jin L: Macrophage polarization in physiological and pathological pregnancy. Front Immunol 10: 792, 2019.

25. Mantovani A, Bottazzi B, Colotta F, Sozzani S and Ruco L: The origin and function of tumor-associated macrophages. Immunol Today 13: 265-270, 1992.

26. Mantovani A and Locati M: Tumor-associated macrophages as a paradigm of macrophage plasticity, diversity, and polarization: Lessons and open questions. Arterioscler Thromb Vasc Biol 33: 1478-1483, 2013.

27. Stein M, Keshav S, Harris N and Gordon S: Interleukin 4 potently enhances murine macrophage mannose receptor activity: A marker of alternative immunologic macrophage activation. J Exp Med 176: 287-292, 1992.

28. Murray PJ, Allen JE, Biswas SK, Fisher EA, Gilroy DW, Goerdt S, Gordon S, Hamilton JA, Ivashkiv LB, Lawrence T, et al: Macrophage activation and polarization: Nomenclature and experimental guidelines. Immunity 41: 14-20, 2014.

29. Qian BZ and Pollard JW: Macrophage diversity enhances tumor progression and metastasis. Cell 141: 39-51, 2010. 
30. Sawa-Wejksza K and Kandefer-Szerszeń M: Tumor-associated macrophages as target for antitumor therapy. Arch Immunol Ther Exp (Warsz) 66: 97-111, 2018.

31. Zhou J, Tang Z, Gao S, Li C, Feng Y and Zhou X: Tumor-Associated Macrophages: Recent insights and therapies. Front Oncol 10: 188, 2020

32. Gratchev A, Guillot P, Hakiy N, Politz O, Orfanos CE, Schledzewski K and Goerdt S: Alternatively activated macrophages differentially express fibronectin and its splice variants and the extracellular matrix protein betaIG-H3. Scand J Immunol 53: 386-392, 2001.

33. Gratchev A, Kzhyshkowska J, Kannookadan S, Ochsenreiter M, Popova A, Yu X, Mamidi S, Stonehouse-Usselmann E, Muller-Molinet I, Gooi L and Goerdt S: Activation of a TGF-beta-specific multistep gene expression program in mature macrophages requires glucocorticoid-mediated surface expression of TGFbeta receptor II. J Immunol 180: 6553-6565, 2008.

34. Goerdt S, Politz O, Schledzewski K, Birk R, Gratchev A, Guillot P, Hakiy N, Klemke CD, Dippel E, Kodelja V and Orfanos CE: Alternative versus classical activation of macrophages. Pathobiology 67: 222-226, 1999.

35. Glass CK and Natoli G: Molecular control of activation and priming in macrophages. Nat Immunol 17: 26-33, 2016.

36. Van Ginderachter JA, Movahedi K, Hassanzadeh Ghassabeh G, Meerschaut S, Beschin A, Raes G and De Baetselier P: Classical and alternative activation of mononuclear phagocytes: Picking the best of both worlds for tumor promotion. Immunobiology 211: 487-501, 2006

37. Osborn O and Olefsky JM: The cellular and signaling networks linking the immune system and metabolism in disease. Nat Med 18: 363-374, 2012.

38. Rőszer T: Understanding the Mysterious M2 macrophage through activation markers and effector mechanisms. Mediators Inflamm 2015: 816460, 2015

39. Tamura R, Tanaka T, Yamamoto Y, Akasaki Y and Sasaki H: Dual role of macrophage in tumor immunity. Immunotherapy 10 : 899-909, 2018.

40. Jinushi M and Komohara Y: Tumor-associated macrophages as an emerging target against tumors: Creating a new path from bench to bedside. Biochim Biophys Acta 1855: 123-1230, 2015.

41. Wynn TA, Chawla A and Pollard JW: Macrophage biology in development, homeostasis and disease. Nature 496: 445-455, 2013.

42. Ginhoux F and Jung S: Monocytes and macrophages: Developmental pathways and tissue homeostasis. Nat Rev Immunol 14: 392-404, 2014

43. Lewis CE, Harney AS and Pollard JW: The multifaceted role of perivascular macrophages in tumors. Cancer Cell 30: 18-25, 2016.

44. Ngambenjawong $\mathrm{CH}$, Heather $\mathrm{H}$ and Suzie $\mathrm{H}$ : Progress in tumor-associated macrophage (TAM)-targeted therapeutics. Adv Drug Deliv Rev 114: 206-221, 2017.

45. Evans R and Alexander P: Cooperation of immune lymphoid cells with macrophages in tumour immunity. Nature 228: 620-622, 1970

46. Chen Y, Song Y, Du W, Gong L, Chang H and Zou Z: Tumor-associated macrophages: An accomplice in solid tumor progression. J Biomed Sci 26: 78, 2019.

47. Jeannin P, Paolini L, Adam C and Delneste Y: The roles of CSFs on the functional polarization of tumor-associated macrophages. FEBS J 285: 680-699, 2018.

48. Wang HW and Joyce JA: Alternative activation of tumor-associated macrophages by IL-4: Priming for protumoral functions. Cell Cycle 9: 4824-4835, 2010.

49. Quail DF and Joyce JA: Microenvironmental regulation of tumor progression and metastasis. Nat Med 19: 1423-1437, 2013.

50. Caux C, Ramos RN, Prendergast GC, Bendriss-Vermare N and Ménétrier-Caux C: A milestone review on how macrophages affect tumor growth. Cancer Res 76: 6439-6442, 2016.

51. Allavena P, Sica A, Solinas G, Porta C and Mantovani A: The inflammatory micro-environment in tumor progression: The role of tumor-associated macrophages. Crit Rev Oncol Hematol 66: $1-9,2008$.

52. Ostuni R, Kratochvill F, Murray PJ and Natoli G: Macrophages and cancer: From mechanisms to therapeutic implications. Trends Immunol 36: 229-239, 2017.

53. Kreider T, Anthony RM, Urban JF Jr and Gause WC: Alternatively activated macrophages in helminth infections. Curr Opin Immunol 19: 448-453, 2007.
54. Wang Q, Ni H, Lan L, Wei X, Xiang R and Wang Y: Fra-1 pro-tooncogene regulates IL-6 expression in macrophages and promotes the generation of M2d macrophages. Cell Res 20: 701-712, 2010.

55. Quail DF and Joyce JA: Molecular pathways: Deciphering mechanisms of resistance to macrophage-targeted therapies. Clin Cancer Res 23: 876-884, 2017.

56. Yuan ZY, Luo RZ, Peng RJ, Wang SS and Xue C: High infiltration of tumor-associated macrophages in triple-negative breast cancer is associated with a higher risk of distant metastasis. Onco Targets Ther 7: 1475-1480, 2014.

57. He Y, Zhang M, Wu X, Sun X, Xu T, He Q and Di W: High MUC2 expression in ovarian cancer is inversely associated with the M1/M2 ratio of tumor-associated macrophages and patient survival time. PLoS One 8: e79769, 2013.

58. Ding P, Wang W, Wang J, Yang Z and Xue L: Expression of tumor-associated macrophage in progression of human glioma. Cell Biochem Biophys 70: 1625-1631, 2014.

59. Pantano F, Berti P, Guida FM, Perrone G, Vincenzi B, Amato MM, Righi D, Dell'Aquila E, Graziano F, Catalano V, et al: The role of macrophages polarization in predicting prognosis of radically resected gastric cancer patients. J Cell Mol Med 17: 1415-1421, 2013.

60. Ruffell B, Affara NI and Coussens LM: Differential macrophage programming in the tumor microenvironment. Trends Immunol 33: 119-126, 2012

61. Mei J, Xiao Z, Guo C, Pu Q, Ma L, Liu C, Lin F, Liao H, You Z and Liu L: Prognostic impact of tumor-associated macrophage infiltration in non-small cell lung cancer: A systemic review and meta-analysis. Oncotarget 7: 34217-34228, 2016.

62. Chanmee T, Ontong P, Konno K and Itano N: Tumor-associated macrophages as major players in the tumor icroenvironment. Cancers (Basel) 6: 1670-1690, 2014.

63. Junankar S, Shay G, Jurczyluk J, Ali N, Down J, Pocock N, Parker A, Nguyen A, Sun S, Kashemirov B, et al: Real-time intravital imaging establishes tumor-associated macrophages as the extraskeletal target of bisphosphonate action in cancer. Cancer Discov 5: 35-42, 2015.

64. Coscia M, Quaglino E, Iezzi M, Curcio C, Pantaleoni F, Riganti C, Holen I, Monkkonen H, Boccadoro M, Forni G, et al: Zoledronic acid repolarizes tumour-associated macrophages and inhibits mammary carcinogenesis by targeting the mevalonate pathway. J Cell Mol Med 14: 2803-2815, 2010.

65. Rogers TL and Holen I: Tumour macrophages as potential targets of bisphosphonates. J Transl Med 9: 177, 2011.

66. Rogers TL, Wind N, Hughes R, Nutter F, Brown HK, Vasiliadou I, Ottewell PD and Holen I: Macrophages as potential targets for zoledronic acid outside the skeleton-evidence from in vitro and in vivo models. Cell Oncol (Dordr) 36: 505-514, 2013.

67. Ali N, Jurczyluk J, Shay G, Tnimov Z, Alexandrov K, Munoz MA, Skinner OP, Pavlos NJ and Rogers MJ: A highly sensitive prenylation assay reveals in vivo effects of bisphosphonate drug on the Rab prenylome of macrophages outside the skeleton. Small GTPases 6: 202-211, 2015.

68. Tardoski S, Ngo J, Gineyts E, Roux JP, Clézardin PH and Melodelima D: Low-intensity continuous ultrasound triggers effective bisphosphonate anticancer activity in breast cancer. Sci Rep 5: 16354, 2015.

69. Sousa S, Auriola S, Mönkkönen J and Maatta J: Liposome encapsulated zoledronate favours M1-like behaviour in murine macrophages cultured with soluble factors from breast cancer cells. BMC Cancer 15: 4, 2015.

70. Hiroshima Y,Maawy A, Hassanein MK, Menen R, Momiyama M, Murakami T, Miwa S, Yamamoto M, Uehara F, Yano S, et al: The tumor-educated-macrophage increase of malignancy of human pancreatic cancer is prevented by zoledronic acid. PLoS One 9: e103382, 2014.

71. Esser AK, Schmieder AH, Ross MH, Xiang J, Su X, Cui G, Zhang H, Yang X, Allen JS, Williams T, et al: Dual-therapy with $\alpha v \beta 3$-targeted $S n 2$ lipase-labile fumagillin-prodrug nanoparticles and zoledronic acid in the $\mathrm{Vx} 2$ rabbit tumor model. Nanomedicine 12: 201-211, 2016.

72. Zekri J, Mansour M and Karim SM: The anti-tumour effects of zoledronic acid. J Bone Oncol 3: 25-35, 2014.

73. Kopecka J, Porto S, Lusa S, Gazzano E, Salzano G, Pinzòn-Daza ML, Giordano A, Desiderio V, Ghigo D, De Rosa G, et al: Zoledronic acid-encapsulating self-assembling nanoparticles and doxorubicin: A combinatorial approach to overcome simultaneously chemoresistance and immunoresistance in breast tumors. Oncotarget 7: 20753-20772, 2016. 
74. Fowler DW, Copier J, Dalgleish AG and Bodman-Smith MD: Zoledronic acid renders human M1 and M2 macrophages susceptible to $\mathrm{V} \delta 2^{+} \gamma \delta \mathrm{T}$ cell cytotoxicity in a perforin-dependent manner. Cancer Immunol Immunother 66: 1205-1215, 2017.

75. Lavin Y and Merad M: Macrophages: Gatekeepers of tissue integrity. Cancer Immunol Res 1: 201-209, 2013.

76. Gul N and van Egmond M: Antibody-dependent phagocytosis of tumor cells by macrophages: A potent effector mechanism of monoclonal antibody. Therapy of cancer. Cancer Res 75: 5008-5013, 2015.

77. Tipton TR, Roghanian A, Oldham RJ, Carter MJ, Cox KL, Mockridge CI, French RR, Dahal LN, Duriez PJ, Hargreaves PG, et al: Antigenic modulation limits the effector cell mechanisms employed by type I anti-CD20 monoclonal antibodies. Blood 125: 1901-1909, 2015.

78. Shi Y, Fan X, Deng H, Brezski RJ, Rycyzyn M, Jordan RE, Strohl WR, Zou Q, Zhang N and An Z: Trastuzumab triggers phagocytic killing of high HER2 cancer cells in vitro and in vivo by interaction with Fcgamma receptors on macrophages. J Immunol 194: 4379-4386, 2015

79. Grugan KD, McCabe FL, Kinder M, Greenplate AR, Harman BC, Ekert JE, van Rooijen N, Anderson GM, Nemeth JA, Strohl WR, et al: Tumor-associated macrophages promote invasion while retaining Fc-dependent anti-tumor function. J Immunol 189: 5457-5466, 2012.

80. Taylor RP and Lindorfer MA: Analyses of CD20 monoclonal antibody-mediated tumor cell killing mechanisms: Rational design of dosing strategies. Mol Pharmacol 86: 485-491, 2014

81. Richards JO, Karki S, Lazar GA, Chen H, Dang W and Desjarlais JR: Optimization of antibody binding to FcgammaRIIa enhances macrophage phagocytosis of tumor cells. Mol Cancer Ther 7: 2517-2527, 2008.

82. Brandsma AM, Ten Broeke T, Nederend M, Meulenbroek LA, van Tetering G, Meyer S, Jansen JH, Beltrán Buitrago MA Nagelkerke SQ, Németh I, et al: Simultaneous Targeting of FcgammaRs and FcalphaRI enhances tumor cell killing. Cancer Immunol Res 3: 1316-1324, 2015.

83. Josephs DH, Bax HJ, Dodev T, Georgouli M, Nakamura M, Pellizzari G, Saul L, Karagiannis P, Cheung A, Herraiz C, et al Anti-folate receptor- $\alpha$ IgE but not IgG recruits macrophages to attack tumors via TNF- $\alpha / \mathrm{MCP}-1$ signaling. Cancer Res 77 $1127-1141,2017$

84. Cespedes MV, Guillen MJ,Lopez-Casas PP, Sarno F, Gallardo A, Álamo P, Cuevas C, Hidalgo M, Galmarini CM, Allavena P, et al: Lurbinectedin induces depletion of tumor-associated macrophages, an essential component of its in vivo synergism with gemcitabine, in pancreatic adenocarcinoma mouse models. Dis Model Mech 9: 1461-1471, 2016.

85. Germano G, Frapolli R, Belgiovine C, Anselmo A, Pesce S Liguori M, Erba E, Uboldi S, Zucchetti M, Pasqualini F, et al: Role of macrophage targeting in the antitumor activity of trabectedin. Cancer Cell 23: 249-262, 2013.

86. Liguori M, Buracchi C, Pasqualini F, Bergomas F, Pesce S, Sironi M, Grizzi F, Mantovani A, Belgiovine C and Allavena P Functional TRAIL receptors in monocytes and tumor-associated macrophages: A possible targeting pathway in the tumor microenvironment. Oncotarget 7: 41662-41676, 2016.

87. De Palma M and Lewis CE: Macrophage regulation of tumor responses to anticancer therapies. Cancer Cell 23: 277-286, 2013

88. Kodumudi KN, Woan K, Gilvary DL, Sahakian E, Wei S and Djeu JY: A novel chemoimmunomodulating property of docetaxel: Suppression of myeloid-derived suppressor cells in tumor bearers. Clin Cancer Res 16: 4583-4594, 2010.

89. Guerriero JL, Ditsworth D, Catanzaro JM, Sabino G, Furie MB, Kew RR, Crawford HC and Zong WX: DNA alkylating therapy induces tumor regression through an HMGB1-mediated activation of innate immunity. J Immunol 186: 3517-3526, 2011.

90. Bryniarski K, Szczepanik M, Ptak M, Zemelka M and Ptak W: Influence of cyclophosphamide and its metabolic products on the activity of peritoneal macrophages in mice. Pharmacol Rep 61: 550-557, 2009.

91. Baghdadi M, Wada H, Nakanishi S, Abe H, Han N, Putra WE, Endo D, Watari H, Sakuragi N, Hida Y, et al: Chemotherapy-Induced IL34 enhances immunosuppression by tumor-associated macrophages and mediates survival of chemoresistant lung cancer cells. Cancer Res 76: 6030-6042, 2016.

92.Zhou Y and Dai Z: New Strategies in the design of nanomedicines to oppose uptake by the mononuclear phagocyte system and enhance cancer therapeutic efficacy. Chem Asian J 13: $3333-3340,2018$
93. Niu M, Naguib YW, Aldayel AM, Shi YC, Hursting SD Hersh MA and Cui Z: Biodistribution and in vivo activities of tumor-associated macrophage-targeting nanoparticles incorporated with doxorubicin. Mol Pharm 11: 4425-4436, 2014.

94. Ngambenjawong C, Cieslewicz M, Schellinger JG and Pun SH: Synthesis and evaluation of multivalent M2pep peptides for targeting alternatively activated M2macrophages. J Control Release 224: 103-111, 2016.

95. Silva VL and Al-Jamal WT: Exploiting the cancer niche: Tumor-associated macrophages and hypoxia as promising synergistic targets for Nano-based therapy. J Control Release 253: 82-96, 2017.

96. Andón FT, Digifico E, Maeda A, Erreni M, Mantovani A, Alonso MJ and Allavena P: Targeting tumor associated macrophages: The new challenge for nanomedicine. Semin Immunol 34: 103-113, 2017.

97. Li M, Zhang F, Su Y, Zhou J and Wang W: Nanoparticles designed to regulate tumor microenvironment for cancer therapy. Life Sci 201: 37-44, 2018

98. Tabata Y and Ikada Y: Effect of the size and surface charge of polymer microspheres on their phagocytosis by macrophage. Biomaterials 9: 356-362, 1988

99. Champion JA and Mitragotri S: Role of target geometry in phagocytosis. Proc Natl Acad Sci USA 103: 4930-4934, 2006

100. He C, Hu Y, Yin L, Tang C and Yin C: Effects of particle size and surface charge on cellular uptake and biodistribution of polymeric nanoparticles. Biomaterials 31: 3657-3666, 2010.

101. Chang YN, Guo H, Li J, Song Y, Zhang M, Jin J, Xing G and Zhao Y: Adjusting the balance between effective loading and vector migration of macrophage vehicles to deliver nanoparticles. PLoS One 8: e76024, 2013.

102. Yu SS, Lau CM, Thomas SN, Jerome WG, Maron DJ, Dickerson JH, Hubbell JA and Giorgio TD: Size- and charge-dependent nonspecific uptake of PEGylated nanoparticles by macrophages. Int J Nanomedicine 7: 799-813, 2012

103. Herd H, Daum N, Jones AT, Huwer H, Ghandehari H and Lehr CM: Nanoparticle geometry and surface orientation influence mode of cellular uptake. ACS Nano 7: 1961-1973, 2013.

104. Li Z, Sun L, Zhang Y, Dove AP, O'Reilly RK and Chen G: Shape effect of Glyco-nanoparticles on macrophage cellular uptake and immune response. ACS Macro Lett 5: 1059-1064, 2016.

105. Cieslewicz M, Tang J, Yu JL, Cao H, Zavaljevski M, Motoyama K, Lieber A, Raines EW and Pun SH: Targeted delivery of proapoptotic peptides to tumor-associated macrophages improves survival. Proc Natl Acad Sci USA 110: 15919-15924, 2013.

106. Ngambenjawong $\mathrm{C}$ and Pun SH: Multivalent polymers displaying M2 macrophage-targeting peptides improve target binding avidity and serum stability. ACS Biomater Sci Eng 3: 2050-2053, 2017.

107. Huang WC, Chen SH, Chiang WH, Huang CW, Lo CL, Chern CS and Chiu HC: Tumor microenvironment-responsive nanoparticle delivery of chemotherapy for enhanced selective cellular uptake and transportation within tumor. Biomacromolecules 17: 3883-3892, 2016.

108. Poupot R, Goursat C and Fruchon S: Multivalent nanosystems: Targeting monocytes/macrophages. Int J Nanomedicine 13: 5511-5521, 2018

109. Cupaioli FA, Zucca FA, Boraschi D and Zecca L: Engineered nanoparticles. How brain friendly is this new guest? Prog Neurobiol 119-120: 20-38, 2014.

110. Costa A, Sarmento B and Seabra V: Mannose-functionalized solid lipid nanoparticles are effective in targeting alveolar macrophages. Eur J Pharm Sci 114: 103-113, 2018.

111. Sarwar HS, Ashraf S, Akhtar S, Sohail MF, Hussain SZ, Rafay M, Yasinzai M, Hussain I and Shahnaz G: Mannosylated thiolated polyethylenimine nanoparticles for the enhanced efficacy of antimonial drug against Leishmaniasis. Nanomedicine (Lond) 13: 25-41, 2018.

112. Fallarini S, Paoletti T, Battaglini CO, Ronchi P, Lay L, Bonomi R, Jha S, Mancin F, Scrimin P and Lombardi G: Factors affecting $T$ cell responses induced by fully synthetic glyco-gold-nanoparticles. Nanoscale 5: 390-400, 2013.

113. He H, Yuan Q, Bie J, Wallace RL, Yannie PJ, Wang J, Lancina MG III, Zolotarskaya OY, Korzun W, Yang $\mathrm{H}$ and Ghosh S: Development of mannose functionalized dendrimeric nanoparticles for targeted delivery to macrophages: Use of this platform to modulate atherosclerosis. Transl Res 193: 13-30, 2018 
114. Sun X, Li W, Zhang X, Qi M, Zhang Z, Zhang XE and Cui Z In vivo targeting and imaging of atherosclerosis using multifunctional virus-like particles of Simian Virus. Nano Lett 16 : 6164-6171, 2016.

115. Zhu S, Niu M, O'Mary H and Cui Z: Targeting of tumor-associated macrophages made possible by PEG-sheddable, mannose-modified nanoparticles. Mol Pharm 10: 3525-3530, 2013.

116. Qian Y, Qiao S, Dai Y, Xu G, Dai B, Lu L, Yu X, Luo Q and Zhang Z: Molecular-targeted immunotherapeutic strategy for melanoma via dual-targeting nanoparticles delivering small interfering RNA to tumor-associated macrophages. ACS Nano 11: 9536-9549, 2017.

117. Lamanna G, Russier J, Dumortier H and Bianco A: Enhancement of anti-inflammatory drug activity by multivalent adamantane-based dendrons. Biomaterials 33: 5610-5617, 2012.

118. Lee S, Kivimae S, Dolor A and Szoka FC: Macrophage-based cell therapies: The long and winding road. J Control Release 240: 527-540, 2016.

119. Choi J, Kim HY, Ju EJ, Jung J, Park J, Chung HK, Lee JS, Lee JS, Park HJ, Song SY, et al: Use of macrophages to deliver therapeutic and imaging contrast agents to tumors. Biomaterials 33 : 4195-4203, 2012.

120.Madsen SJ, Baek SK, Makkouk AR, Krasieva T and Hirschberg H: Macrophages as cell-based delivery systems for nanoshells in photothermal therapy. Ann Biomed Eng 40: 507-515, 2012.

121. Ikehara Y, Niwa T, Biao L, Ikehara SK, Ohashi N, Kobayashi T, Shimizu Y, Kojima N and Nakanishi H: A carbohydrate recognition-based drug delivery and controlled release system using intraperitoneal macrophages as a cellular vehicle. Cancer Res 66: 8740-8748, 2006.

122. Miller MA, Zheng YR, Gadde S, Pfirschke C, Zope H, Engblom C, Kohler RH, Iwamoto Y, Yang KS, Askevold B, et al: Tumour-associated macrophages act as a slow-release reservoir of nano-therapeutic Pt(IV) pro-drug. Nat Commun 6: 8692, 2015.
123. Tanei T, Leonard F, Liu X, Alexander JF, Saito Y, Ferrari M, Godin B and Yokoi K: Redirecting transport of nanoparticle albumin-bound paclitaxel to macrophages enhances therapeutic efficacy against liver metastases. Cancer Res 76: 429-439, 2016.

124. Dou H, Destache CJ, Morehead JR, Mosley RL, Boska MD, Kingsley J, Gorantla S, Poluektova L, Nelson JA, Chaubal M, et al: Development of a macrophage-based nanoparticle platform for antiretroviral drug delivery. Blood 108: 2827-2835, 2006

125. Dou H, Grotepas CB, McMillan JM, Destache CJ, Chaubal M, Werling J, Kipp J, Rabinow B and Gendelman HE: Macrophage delivery of nanoformulated antiretroviral drug to the brain in a murine model of neuroAIDS. J Immunol 183: 661-669, 2009.

126. Zhao Y, Haney MJ, Klyachko NL, Li S, Booth SL, Higginbotham SM, Jones J, Zimmerman MC, Mosley RL, Kabanov AV, et al: Polyelectrolyte complex optimization for macrophage delivery of redox enzyme nanoparticles. Nanomedicine (Lond) 6: 25-42, 2011.

127. Klyachko NL, Haney MJ, Zhao Y, Manickam DS, Mahajan V, Suresh P, Hingtgen SD, Mosley RL, Gendelman HE, Kabanov AV and Batrakova EV: Macrophages offer a paradigm switch for CNS delivery of therapeutic proteins. Nanomedicine (Lond) 9: 1403-1422, 2014.

128. Muthana M, Kennerley AJ, Hughes R, Fagnano E, Richardson J, Paul M, Murdoch C, Wright F, Payne C, Lythgoe MF, et al: Directing cell therapy to anatomic target sites in vivo with magnetic resonance targeting. Nat Commun 6: 8009, 2015.

129. Han J, Zhen J, Du Nguyen V, Go G, Choi Y, Ko SY, Park JO and Park S: Hybrid-actuating macrophage-based microrobots for active cancer therapy. Sci Rep 6: 28717, 2016. 